\title{
Efeito do Método Pilates na dor lombar: revisão integrativa
}

\author{
Effect of the Pilates Method in low back pain: integrative review
}

\section{Bruno Moro Dal Bem \\ Deise Iop Tavares ${ }^{2}$ \\ Alecsandra Pinheiro Vendrusculo ${ }^{3}$}

\section{Endereço para correspondência:}

Alecsandra Pinheiro Vendrusculo

Rua Felipe dos Santos, 66, Bairro Duque de Caxias

97070-340 - Santa Maria, RS [Brasil]

alec@ufn.edu.br
1 Departamento de Fisioterapia, Universidade Franciscana UFN. Santa Maria, RS - Brasil.

ORCID: https://orcid.org/0000-0001-8145-129X

2 Departamento do Programa de Pós-Graduação em Gerontologia, Universidade Federal de Santa Maria - UFSM. Santa Maria, RS - Brasil.

ORCID: https://orcid.org/0000-0002-7467-226X

3 Departamento de Fisioterapia, Universidade Franciscana UFN. Santa Maria, RS - Brasil.

ORCID: https://orcid.org/0000-0002-6858-0094

\begin{abstract}
Resumo
Introdução: A dor lombar é uma das patologias que acomete uma grande parcela da população, e a procura do Método Pilates (MP) relacionado a ela tem aumentado exponencialmente. Objetivos: Realizar uma revisão integrativa sobre a utilização do MP pelo fisioterapeuta na dor lombar. Métodos: As bases de dados utilizadas foram PUBMED e SCIELO, foram aplicados os critérios de inclusão e exclusão, após a aplicação destes, restaram 14 estudos. Resultados: A presente pesquisa mostrou que um programa de Pilates para a dor lombar gera diversos efeitos positivos no tratamento, trazendo como benefícios a diminuição da dor e da incapacidade funcional, bem como aumento da flexibilidade e força muscular de indivíduos, além de ocasionar melhora na qualidade de vida e na diminuição do medo de praticar exercício físico. Conclusão: O MP pode ser utilizado pelo fisioterapeuta para diversos fins, como no tratamento da dor lombar, trazendo assim benefícios na diminuição da dor, na redução da incapacidade funcional além da melhora da qualidade de vida.
\end{abstract}

Descritores: Dor Lombar. Técnicas de Exercício e de Movimento. Fisioterapia.

\begin{abstract}
Introduction: Lumbar pain is one of the pathologies that is affecting the population today and also in the search for the Pilates Method (MP) related to it. Objectives: To perform an integrative review on the use of PM by the physiotherapist in low back pain. Methods: The databases used were PUBMED and SCIELO, the inclusion and exclusion criteria were applied, after the application of these, 14 studies remained. Results: The present research showed that a Pilates program for low back pain generates several positive effects in the treatment, bringing as benefits the reduction of pain and the functional incapacity as well as increase of the flexibility and muscular strength of individuals, besides causing improvement in the quality of life and in reducing the fear of practicing physical exercise. Conclusion: The MP can be used by the physiotherapist for several purposes, such as in the treatment of low back pain, thus bringing benefits in reducing pain, reducing functional disability and improving quality of life.
\end{abstract}

Keywords: Low Back Pain. Exercise and Movement Techniques. Physical Therapy. 


\section{Introdução}

O Método Pilates (MP) foi criado por Joseph Humbertus Pilates, nascido em 1880, na Alemanha, que sofria de diversas doenças, como asma, raquitismo e febre reumática ${ }^{1}$. Devido a isso, em meados da Primeira Guerra Mundial, desenvolveu o método, que se baseava em movimentos utilizados em ginástica, yoga, dança e artes marciais. Com esse conjunto de movimentos combinados, conseguiu desenvolver uma série de atividades que focava no corpo como um todo, e que tinha como objetivo principal fortalecer musculaturas do abdômen e paravertebrais, além do ganho de flexibilidade ${ }^{2}$.

Primeiramente, o método foi testado e utilizado em camas de hospitais por meio de molas, para adquirir força, resistência e condicionamento físico de pessoas hospitalizadas. Posteriormente, veio a ser utilizado para diversos fins ${ }^{1}$.

O método é baseado em alguns princípios básicos, como concentração, centro de força (Power House), controle, precisão, fluxo e respiração, sendo este considerado o principal durante a execução do exercício. O método pode ser realizado em diversas posições, como no chão sobre um tapete, que serve para evitar impactos excessivos e pressões sobre os músculos, articulações e tecidos. Outra forma de realizar é em aparelhos, como Reformer, Cadillac, Ladder Barrel, Step Chair, que são equipamentos com uso de molas e polias ${ }^{3,4,5}$. O MP vem sendo cada vez mais procurado para prevenir e reabilitar lesões, melhorar o condicionamento físico, aumentar a flexibilidade muscular, reduzir o estresse, tratar alterações posturais, entre outras. Com isso, torna-se um grande coadjuvante na reabilitação e uma opção de modalidade de exercício físico ${ }^{6}$.

A dor lombar é uma das patologias que vem se mostrando com frequência na população, e a procura do MP relacionado a ela vem aumentando. Existem maneiras de classificá-la, como dor específica e inespecífica e também quanto à duração, que pode ser aguda, subaguda ou crônica. Além disso, a lombalgia é uma das principais causas de incapacidade funcional, e, consequentemente, causa a redução da capacidade de resistência à fadiga dos músculos extensores do tronco e a ineficiência do recrutamento muscular dos estabilizadores profundos do tronco ${ }^{7}$.

A fisioterapia, nos últimos anos, vem aumentando a gama de tratamentos com a utilização do MP. Assim, torna-se uma ferramenta para reabilitar diversas lesões e patologias por meio de séries de exercícios que variam os níveis de dificuldade e que podem ser adaptados conforme a habilidade e limitação de cada indivíduo, além de poder ser realizado por todas as faixas etárias, fazendo-se um adjunto na reabilitação, bem como na manutenção da saúde física ${ }^{5,8}$.

O objetivo da presente pesquisa foi realizar uma revisão integrativa sobre a utilização do MP pelo fisioterapeuta na dor lombar.

\section{Materiais e métodos}

Trata-se de uma revisão integrativa, que é a mais abrangente abordagem metodológica relacionada às revisões, incluindo pesquisas experimentais e não-experimentais. Além disso, essa revisão emerge com uma metodologia que possibilita a síntese do conhecimento e a incorporação da aplicabilidade de resultados de estudos significativos na prática9.

As palavras-chave utilizadas no presente estudo foram as seguintes: Pilates, Fisioterapia, Dor Lombar, Physiotherapy, physical therapy, low back pain, usando as bases de dados, PUBMED e SCIELO, e ainda entrecruzaram-se utilizando operador boleano and.

Foram considerados como critérios de inclusão para a realização do estudo os artigos que estavam disponíveis na íntegra e publicados entre os anos de 2012 e 2017, relacionados ao $\mathrm{MP}$, à fisioterapia e à dor lombar, nos idiomas português e inglês. Já os critérios de exclusão fo- 
ram somente estudos que não fossem obtidos na íntegra, que não foram realizados por fisioterapeutas e artigos duplicados.

Os estudos selecionados para a presente pesquisa foram apresentados de forma descrita e listados em forma de quadros, apresentando autor/ano, amostra, desenho do estudo, intervenção e resultados. No seguimento, os dados foram confrontados com a literatura para melhor elucidação do uso do Método Pilates pelo fisioterapeuta na dor lombar.

\section{Resultados}

Ao realizar a seleção do material a ser utilizado na presente pesquisa, verificou-se um significativo número de estudos que utilizam o MP. Devido a isso, optou-se por realizar a apresentação dos resultados em forma de fluxograma e quadros para melhor entendimento dos leitores.

Na figura 1, está descrito o fluxograma que demonstra como foi realizada a seleção dos dados e o número de estudos encontrados após a busca realizada nas bases de dados PUBMED e SCIELO. As palavras-chave foram entrecruzadas e, após a aplicação dos critérios de inclusão e exclusão, chegou-se a um total de 14 artigos, sendo 13 em inglês e um em português.

Os estudos selecionados foram agrupados por autoria e ano, amostra, desenho do estudo, intervenção e resultados, conforme descritos nos quadros 1, 2, 3 e 4 .

\section{Discussão}

No presente estudo, foi realizada uma revisão integrativa, abordando estudos sobre a utilização do MP pelo fisioterapeuta na dor lombar, e na maioria, abordaram-se aspectos de incapacidade funcional e equilíbrio, que estão relacionados à dor.

Da Silva e Mannrich (2009) ${ }^{10}$ pesquisaram sobre as finalidades do MP e encontraram, por intermédio de uma revisão sistemática, que, além do ganho de força muscular, que era o objetivo inicial do método, hoje também se utiliza para lombalgia, correção postural, ganho de massa óssea, ganho de força no período pósoperatório, mas, principalmente, no tratamento da lombalgia.

Outro benefício do MP é a melhora do condicionamento respiratório, que acontece devido ao estímulo da função do sistema respiratório durante a realização dos exercícios. Esse estímulo acaba levando ao aumento da Pressão Expiratória Máxima (PEM), o que confirma a indicação do MP para esse benefício, em especial na população idosa ${ }^{11}$.

Gradativamente, o MP vem se mostrando eficaz na dor lombar, e, de acordo com Wells et al. (2014) ${ }^{12}$, há um consenso total entre os fisioterapeutas em relação à indicação, aos benefícios e às precauções do método sobre a dor lombar. $\mathrm{O}$ estudo de Wells et al. corrobora o de Duarte, Sousa e Nunes, (2017) ${ }^{13}$, que também encontraram benefícios do MP na condição física e de saúde,
Figura 1: Fluxograma sobre metodologia da seleção dos estudos encontrados na presente pesquisa.

Fonte: Autores, Santa Maria, 2018. 


\begin{tabular}{|c|c|c|c|c|}
\hline Autor/Ano & Amostra & Desenho do Estudo & Intervenção & Resultados \\
\hline $\begin{array}{l}\text { Miyamoto et al., } \\
\text { (2012) }\end{array}$ & $\begin{array}{l}0 \text { estudo teve uma amostra } \\
\text { de } 86 \text { participantes com dor } \\
\text { lombar crônica não específica } \\
\text { com duração de pelo menos } \\
\text { três meses e idade entre } 18 \text { a } \\
60 \text { anos. }\end{array}$ & $\begin{array}{l}\text { Estudo randomizado } \\
\text { controlado e aleatório. }\end{array}$ & $\begin{array}{l}\text { Os pacientes selecionados } \\
\text { receberam um folheto de } \\
\text { educação, contendo informações } \\
\text { sobre dor nas costas, e foram } \\
\text { alocados aleatoriamente para } \\
\text { receber } 12 \text { sessões de exercícios } \\
\text { com base nos princípios } \\
\text { do Pilates ao longo de seis } \\
\text { semanas ( } n=43 \text { ) ou não (ou seja, } \\
\text { educação sozinho) ( } n=43 \text { ). }\end{array}$ & $\begin{array}{l}\text { A adição de Pilates e um livreto } \\
\text { educacional ofereceu pequenos } \\
\text { benefícios em comparação com } \\
\text { a educação de pacientes. E ainda } \\
\text { notaram melhoras em relação à } \\
\text { dor, incapacidade funcional no } \\
\text { Grupo Pilates após intervenção. } \\
\text { Porém essas diferenças } \\
\text { não foram estatisticamente } \\
\text { significativas aos seis meses. }\end{array}$ \\
\hline $\begin{array}{l}\text { Luz Junior et al. } \\
\qquad(2013)\end{array}$ & $\begin{array}{c}\text { Foi composta por } 86 \text { pacientes } \\
\text { que procuraram atendimento } \\
\text { de dor lombar crônica não } \\
\text { específica, com duração de } \\
\text { sintomas de pelo menos três } \\
\text { meses e idade entre } 18 \text { e } 60 \\
\text { anos. }\end{array}$ & $\begin{array}{l}\text { Trata-se de um estudo } \\
\text { controlado randomizado } \\
\text { com um avaliador cego. } \\
\text { que visa avaliar a eficácia } \\
\text { dos exercícios de Pilates } \\
\text { baseados em Mat e } \\
\text { Equipamentos do Pilates, em } \\
\text { pacientes com dor lombar } \\
\text { crônica não específica. }\end{array}$ & $\begin{array}{l}\text { Avaliar a eficácia dos exercícios } \\
\text { de Pilates baseados em Mat e } \\
\text { Equipamentos, em pacientes } \\
\text { com dor lombar crônica não } \\
\text { específica. Foram realizadas } \\
\text { sessões durante uma hora com } \\
\text { frequência de duas vezes por } \\
\text { semana, durante um período de } \\
\text { seis semanas. }\end{array}$ & $\begin{array}{l}\text { Verificou-se que não há } \\
\text { diferença entre os métodos na } \\
\text { diminuição da dor lombar, pois } \\
\text { os dois foram eficientes para tal } \\
\text { objetivo. }\end{array}$ \\
\hline $\begin{array}{l}\text { Marshall et al. } \\
(2013)\end{array}$ & $\begin{array}{l}\text { A amostra foi composta por } 64 \\
\text { participantes com dor lombar, } \\
\text { divididos aleatoriamente em } \\
\text { dois grupos. } 0 \text { grupo específico } \\
\text { de exercício do tronco (SEG), } \\
\text { que realizou Pilates, e o grupo } \\
\text { de ciclismo estacionário (CEG). }\end{array}$ & Estudo controlado aleatório. & $\begin{array}{l}\text { Realizados exercícios três vezes } \\
\text { por semana, durante um total } \\
\text { de oito semanas. Avaliaram os } \\
\text { participantes depois de oito } \\
\text { semanas de tratamento e após } \\
\text { seis meses. }\end{array}$ & $\begin{array}{l}\text { Após oito semanas, verificou-se } \\
\text { que a incapacidade foi } \\
\text { significativamente menor no } \\
\text { grupo SEG. A dor lombar foi } \\
\text { reduzida em ambos os grupos. } \\
\text { Em longo prazo, não se notou } \\
\text { diferença entre os grupos. }\end{array}$ \\
\hline
\end{tabular}

Quadro 1: Estudos selecionados para a presente pesquisa após aplicação dos critérios de inclusão e exclusão

Fonte: Autores, Santa Maria, 2018.

\begin{tabular}{|c|c|c|c|c|}
\hline Autor/Ano & Amostra & Desenho do Estudo & Intervenção & Resultados \\
\hline $\begin{array}{l}\text { Miyamoto, Costa e } \\
\text { Cabral (2013) }\end{array}$ & $\begin{array}{c}\text { A amostra foi composta por } \\
\text { oito artigos. }\end{array}$ & $\begin{array}{l}\text { Revisão sistemática e } \\
\text { meta-análise. }\end{array}$ & & $\begin{array}{l}0 \text { MP foi mais eficaz em relação } \\
\text { a uma intervenção mínima para } \\
\text { redução da dor e incapacidade } \\
\text { em pacientes com dor lombar, } \\
\text { porém, somente em curto prazo } \\
\text { de tratamento. }\end{array}$ \\
\hline Wells et al. (2013). & $\begin{array}{l}\text { Este estudo examinou cinco } \\
\text { revisões sistemáticas que } \\
\text { investigaram a eficácia } \\
\text { do exercício de Pilates em } \\
\text { pessoas com dor lombar } \\
\text { crônica. }\end{array}$ & Revisão sistemática. & & $\begin{array}{c}\text { Concluiu-se que o Pilates não foi } \\
\text { eficaz na redução da dor devido } \\
\text { ao baixo número de artigos e da } \\
\text { qualidade destes. }\end{array}$ \\
\hline $\begin{array}{l}\text { Mostagi et al. } \\
\quad(2014)\end{array}$ & $\begin{array}{c}\text { Foram recrutados } 22 \\
\text { participantes, com idades } \\
\text { entre } 18 \text { e } 55 \text { anos. No total, } 11 \\
\text { participantes no Grupo Pilates } \\
\text { (PG) e } 11 \text { participantes para } \\
\text { o (Grupo de exercícios gerais } \\
\text { (GEG). }\end{array}$ & $\begin{array}{l}\text { Estudo randomizado e } \\
\text { controlado. }\end{array}$ & $\begin{array}{l}\text { Os participantes realizaram } \\
\text { um total de } 16 \text { sessões, duas } \\
\text { vezes por semana, durante oito } \\
\text { semanas. Para ambos os grupos, } \\
\text { as intervenções foram no caráter } \\
\text { individual/privado de uma hora. }\end{array}$ & $\begin{array}{l}\text { Ambos os grupos foram } \\
\text { semelhantes em todas as } \\
\text { características avaliadas. No } \\
\text { entanto, no grupo GEG, houve } \\
\text { melhora estatisticamente na } \\
\text { funcionalidade e flexibilidade. } 0 \\
\text { PG não apresentou diferenças. }\end{array}$ \\
\hline $\begin{array}{l}\text { Vasconcellos et al. } \\
\text { (2014) }\end{array}$ & Composta por 47 estudos. & Revisão sistemática. & & $\begin{array}{l}\text { Contatou-se desequilíbrio } \\
\text { entre os músculos agonistas- } \\
\text { antagonistas do tronco. A } \\
\text { ativação ineficaz do transverso } \\
\text { abdominal é fator de risco para a } \\
\text { dor lombar. }\end{array}$ \\
\hline
\end{tabular}

Quadro 2: Estudos selecionados para a presente pesquisa após aplicação dos critérios de inclusão e exclusão

Fonte: Autores, Santa Maria, 2018. 


\begin{tabular}{|c|c|c|c|c|}
\hline Autor/Ano & Amostra & Desenho do Estudo & Intervenção & Resultados \\
\hline Wells et al. (2014) & $\begin{array}{c}\text { Foram registrados } 30 \\
\text { Fisioterapeutas no Conselho de } \\
\text { Fisioterapia da Austrália para } \\
\text { que tratassem pessoas com } \\
\text { CLBP com o Pilates e capazes } \\
\text { de completar três rodadas no } \\
\text { mínimo da pesquisa Delphi. }\end{array}$ & Pesquisa Delphi & $\begin{array}{l}\text { Foram enviados três questioná- } \\
\text { rios aos participantes entre março } \\
\text { e junho de } 2012.0 \text { consenso } \\
\text { foi definido quando } 70 \% \text { dos } \\
\text { participantes concordavam } \\
\text { ou discordavam de um item. A } \\
\text { força do acordo ou desacordo foi } \\
\text { avaliada por meio da classificação } \\
\text { das respostas com uma escala de } \\
\text { resposta Likert. }\end{array}$ & $\begin{array}{l}\text { Foram encontrados um consenso } \\
\text { de } 100 \% \text { conforme benefícios, } \\
\text { indicações e precauções. } \\
50 \% \text { sobre riscos e } 56 \% \text { de } \\
\text { contraindicações. Notaram } \\
\text { que o Pilates pode melhorar a } \\
\text { capacidade funcional, confiança } \\
\text { do movimento, consciência } \\
\text { corporal, postura e controle de } \\
\text { movimentos. }\end{array}$ \\
\hline $\begin{array}{l}\text { Cruz-Díaz et al. } \\
\qquad(2015)\end{array}$ & $\begin{array}{c}\text { A amostra foi composta por } \\
103 \text { mulheres com faixa etária } \\
\text { maior de } 65 \text { anos. }\end{array}$ & $\begin{array}{l}\text { Ensaio controlado aleatório } \\
\text { único e cego. }\end{array}$ & $\begin{array}{l}\text { Os pacientes foram alocados } \\
\text { aleatoriamente, para grupo } \\
\text { fisioterapia e Pilates (PPTG) } \\
\text { ou grupo fisioterapia (PTG). Os } \\
\text { grupos receberam intervenção } \\
\text { durante seis semanas, duas } \\
\text { vezes por semana. }\end{array}$ & $\begin{array}{l}\text { Os resultados obtidos sugerem a } \\
\text { adição do Pilates à fisioterapia, } \\
\text { pois diminui o medo de queda e } \\
\text { aumenta o equilíbrio funcional, } \\
\text { aspecto que pode estar ligado à } \\
\text { dor lombar e ao risco de quedas } \\
\text { em mulheres acima de } 65 \text { anos. }\end{array}$ \\
\hline $\begin{array}{l}\text { Cruz-Díaz et al. } \\
\qquad(2015)\end{array}$ & $\begin{array}{l}\text { A amostra foi composta por } \\
123 \text { pacientes com história de } \\
\text { pelo menos } 12 \text { semanas de dor } \\
\text { lombar (LBP); mulheres idade } \\
\text { entre } 45 \text { e } 75 \text { anos, que vinham } \\
\text { sofrendo há pelo menos três } \\
\text { meses com dores lombares. }\end{array}$ & $\begin{array}{l}\text { Ensaio clínico controlado } \\
\text { aleatório único. }\end{array}$ & $\begin{array}{c}\text { Foram divididos em grupo Pilates } \\
\text { e Fisioterapia. Foi seguido } \\
\text { protocolo de duas vezes por } \\
\text { semana, durante seis semanas } \\
\text { e grupo Fisioterapia, que utilizou } \\
\text { aplicação de eletroterapia e } \\
\text { mobilização articular da coluna } \\
\text { lombar. }\end{array}$ & $\begin{array}{l}\text { Não foram encontradas } \\
\text { diferenças significativas } \\
\text { entre os grupos para todas as } \\
\text { variáveis dependentes (dor e } \\
\text { incapacidade). }\end{array}$ \\
\hline
\end{tabular}

Quadro 3: Estudos selecionados para a presente pesquisa após aplicação dos critérios de inclusão e exclusão

Fonte: Autores, Santa Maria, 2018.

incluindo flexibilidade, qualidade de vida, capacidade funcional, entre outros.

Guimarães et al. $(2014)^{14}$, que realizaram um estudo experimental com objetivo de verificar o efeito do Método Pilates no nível de flexibilidade de quadril e cintura escapular de idosos, notaram que, no decorrer do estudo, idosas que praticaram Pilates tiveram um aumento na flexibilidade, além de prevenir que ela piore com o avanço da idade. Diante disso, a prática do Pilates é aconselhada para esse público.

Em procedência da diversidade de estudos entre MP e somente fisioterapia, houve um estudo de caso atual realizado por Ribeiro, Oliveira e Blois, $(2015)^{15}$, que dividiram os participantes em Grupo de Cinesioterapia (GC) e Grupo Pilates (GP) e perceberam que, no GP, a incapacidade funcional reduziu estatisticamente $(p<0,042)$. Porém, em uma pesquisa de Wajswelner, Metcalf e Behfnell (2012) ${ }^{16}$, que foi um estudo piloto, no qual também foram separados em Grupo Pilates e Grupo de exercícios gerais, os resultados mostraram que, em relação com a Escala Quebec, que avalia a incapacidade funcional, não ocorreu diferença entre ambos os grupos.

Ainda existem debates sobre a aplicação do MP e a fisioterapia convencional na dor lombar e qual sua eficácia. Yamatto et al. (2016) ${ }^{17}$ fizeram uma revisão sistemática para uma comparação do Pilates com outras atividades comuns e perceberam que não houve diferenças significativas, a não ser em relação à dor, que diminuiu em curto prazo. Em contrapartida, Galdino et al. $(2015)^{18}$ realizaram uma revisão na qual encontraram resultados positivos relacionados à eficácia e aos benefícios do MP aplicados na dor lombar crônica não específica, agindo na redução da dor e incapacidade funcional.

O MP vem atuando, na maioria das vezes, quando a dor e instabilidade já estão instaladas no indivíduo. Por isso, o método tem papel importante para combater a dor, pois trabalha 


\begin{tabular}{|c|c|c|c|c|}
\hline Autor/Ano & Amostra & Desenho do Estudo & Intervenção & Resultados \\
\hline $\begin{array}{c}\text { Ribeiro, Oliveira } \\
\text { e Blois (2015) }\end{array}$ & $\begin{array}{c}\text { A amostra foi composta } \\
\text { por cinco pacientes com } \\
\text { dor lombar (três mulheres } \\
\text { e dois homens, idade } \\
\text { média de } 32,4 \pm 15,6 \\
\text { anos). }\end{array}$ & Estudo de caso. & $\begin{array}{c}\text { Foram divididos em dois } \\
\text { grupos e submetidos a } 20 \\
\text { sessões de tratamento, } \\
\text { duas vezes por semana. O } \\
\text { grupo A realizou o protocolo } \\
\text { de Cinesioterapia e o grupo } \\
\text { B, exercícios do MP. }\end{array}$ & $\begin{array}{c}\text { A análise de ambos os } \\
\text { métodos revelou uma } \\
\text { redução estatisticamente } \\
\text { significativa na incapaci- } \\
\text { dade funcional, consequen- } \\
\text { temente no quadro da dor } \\
(p=0,042) \text {. }\end{array}$ \\
\hline $\begin{array}{c}\text { Stieglitz, Vinson } \\
\text { e Hampton } \\
(2015)\end{array}$ & $\begin{array}{c}\text { Completaram o estudo } 12 \\
\text { participantes, com idades } \\
\text { entre } 20 \text { e } 65 \text { anos e que } \\
\text { não tivessem experiência } \\
\text { com MP. }\end{array}$ & Estudo Piloto. & \begin{tabular}{|} 
Os 12 participantes \\
sofriam dor e incapacidade \\
funcional devido ao \\
trabalho. Foram avaliados \\
pré e pós o exercício do \\
MP em equipamentos \\
durante seis semanas.
\end{tabular} & $\begin{array}{l}\text { A intervenção reduziu } \\
\text { significativamente a dor, } \\
\text { de acordo com a VAS } \\
(p<0,0001) \text { e a incapaci- } \\
\text { dade funcional de acordo } \\
\text { com o ODI }(p<0,02) \text {. }\end{array}$ \\
\hline $\begin{array}{l}\text { Patti et al. } \\
\text { (2016) }\end{array}$ & $\begin{array}{l}\text { Trinta e oito participantes } \\
\text { foram escolhidos e } \\
\text { divididos em grupos, o } \\
\text { experimental (EG) e de } \\
\text { controle (CG). }\end{array}$ & $\begin{array}{c}\text { Ensaio Randomizado e } \\
\text { controlado. }\end{array}$ & $\begin{array}{l}\text { As aulas eram de } 50 \\
\text { minutos, seguindo um } \\
\text { protocolo de Pilates } \\
\text { pré-estabelecido. O EG foi } \\
\text { dividido em duas classes, e } \\
\text { a intervenção foi realizada } \\
\text { três vezes por semana. } \\
\text { Todos os exercícios foram } \\
\text { completados realizados em } \\
\text { dois níveis de dificuldade: } \\
\text { básico e intermediário. }\end{array}$ & $\begin{array}{l}\text { As medidas de posturo- } \\
\text { grafia melhoraram para os } \\
\text { participantes no EG, com } \\
\text { ambos os olhos abertos } \\
\text { e os olhos fechados ( } P \\
<0,05 \text { ). A dor diminuiu } \\
\text { significativamente em } \\
\text { ambos os grupos ao } \\
\text { longo das } 14 \text { semanas do } \\
\text { protocolo de estudo. }\end{array}$ \\
\hline $\begin{array}{l}\text { Yamato et al. } \\
\qquad(2016)\end{array}$ & $\begin{array}{l}\text { Incluídos nesta revisão } \\
\text { somente } 10 \text { estudos. }\end{array}$ & Revisão Sistemática. & & $\begin{array}{l}\text { Verificou-se que não houve } \\
\text { diferença significativa entre } \\
\text { MP e outras atividades. No } \\
\text { entanto, em curto e médio } \\
\text { prazo reduziu a dor e a } \\
\text { incapacidade funcional. }\end{array}$ \\
\hline
\end{tabular}

Quadro 4: Estudos selecionados para a presente pesquisa após aplicação dos critérios de inclusão e exclusão.

Fonte: Autores, Santa Maria, 2018.

exercícios que visam ao equilíbrio entre força, flexibilidade e resistência do corpo ${ }^{19}$.

Guclu-Gunduz et al. $(2014)^{20}$ realizaram um estudo sobre o efeito do MP em pacientes com Esclerose Múltipla (EM) e mostraram que um programa de exercícios de oito semanas tem grande benefício no equilíbrio, na mobilidade e na força de indivíduos que apresentam EM. Já Tomruk et al. (2016) ${ }^{21}$ fizeram um estudo semelhante e constataram que, em 10 semanas, o Pilates apresentou diferença significativa no controle postural e na diminuição da fadiga dos indivíduos com EM. Além disso, demonstraram que o MP é benéfico e seguro para ser usado na fase ambulatorial.

Nota-se que o MP pode ser utilizado para diversos tratamentos, como na Osteoporose, segundo Angin, Erden e Can, (2015)22, que realiza- ram um estudo sobre o efeito do Pilates na densidade mineral óssea (DMO), no desempenho físico e na qualidade de vida $(\mathrm{QV})$ de mulheres pós-menopausa. Com esse estudo, perceberam um resultado significativo sobre o aumento da $\mathrm{DMO}$, do desempenho físico e da QV no grupo pilates $(\mathrm{p}<0,05)$.

Houve um estudo realizado por Bird e Fell, $(2014)^{23}$, que compararam o efeito do MP sobre o equilíbrio na pré-intervenção, pós-intervenção e 12 meses depois. E notaram que, após 12 meses da intervenção, houve diferença significativa no equilíbrio dinâmico daqueles indivíduos que continuaram com o Pilates.

Após o descrito, verificou-se que o MP vem sendo muito utilizado pelos fisioterapeutas no tratamento de diversas patologias, entre elas, a dor lombar. 


\section{Conclusão}

Com esta revisão, verificou-se que o MP pode ser utilizado pelo fisioterapeuta para diversos fins, como no tratamento da dor lombar, mostrando-se mais uma ferramenta importante para diversificar e agregar no tratamento da dor lombar. Porém, em diversos momentos, não se apresentou mais eficaz do que outras modalidades de tratamento.

Com este estudo, percebeu-se que um programa de Pilates para a dor lombar gera efeitos positivos no tratamento, pois traz benefícios na diminuição da dor, na redução da incapacidade funcional, no aumento da flexibilidade e força muscular de indivíduos. Além disso, melhora a qualidade de vida e diminui o medo de praticar exercício físico.

\section{Referências}

1. Marés G, De Oliveira KB, Piazza MC, Preis C, Neto LB. A importância da estabilização central no método Pilates: uma revisão sistemática. Fisioter. mov. 2017;25 (2):445-451.

2. Silva MAC, Dias JM, Silva MF, Mazuquin BF, Abrão T, Cardoso JR. Análise comparativa da atividade elétrica do músculo multífido durante exercícios do Pilates, série de Williams e Spine Stabilization. Fisioter. mov. 2017;26 (1):87-94.

3. Sarmento LA, Pinto JS, da Silva AP, Cabral CM, Chiavegato LD. Effect of conventional physical therapy and Pilates in functionality, respiratory muscle strength and ability to exercise in hospitalized chronic renal patients: a randomized controlled trial. Clin. Rehabil. 2017;31 (4):508-520.

4. Natour J, Cazotti LDA, Ribeiro LH, Baptista AS, Jones A. Pilates improves pain, function and quality of life in patients with chronic low back pain: a randomized controlled trial. Clin. Rehabil. 2015;29 (1):59-68.

5. Da Luz MAJ, Costa LOP, Fuhro FF, Manzoni ACT, Oliveira NTB, Cabral CMN. Effectiveness of mat Pilates or equipment-based Pilates exercises in patients with chronic nonspecific low back pain: a randomized controlled trial. Braz. J. Phys. Ther. 2014;94 (5):623-631.
6. Do Prado Macêdo ÉM, das Neves SF, Palma MA, Motta-Santos D, Rauber SB, Brandão PS. Efeito de sessão aguda de Pilates no solo e na água sobre a glicemia de mulheres portadoras de diabetes tipo 2 . Rev. Bras. Fisioter. 2017;18 (1):47-55

7. Silveira APB, Nagel LZ, Pereira DD, Morita AK, Spinoso DH, Navega MT et al. Efeito agudo de exercícios do método Pilates na ativação dos músculos do tronco de pessoas com e sem dor lombar. Conscientiae Saúde. 2016;15 (2):231-240.

8. Stivala A, Hartley G. The effects of a Pilates-based exercise rehabilitation program on functional outcome and fall risk reduction in an aging adult status-post traumatic hip fracture due to a fall. J Geriatr Phys Ther. 2014;37 (3):136-145.

9. De Souza, MT, da Silva MD, de Carvalho R. Revisão integrativa: o que é e como fazer. Einstein. 2010;8, p.102-6.

10. Da Silva ACLG, Mannrich G. Pilates na reabilitação: uma revisão sistemática. Fisioter. Mov. 2009;22 (3):449-455

11. Lopes EDS, Ruas G, Patrizzi LJ. Efeitos de exercício do Método Pilates na força muscular respiratória de idosas: um ensaio clínico. Rev. Bras. Geriatr. Gerontol. 2014;17 (3):517-523.

12. Wells C, Kolt GS, Marshall P, Bialocerkowski A. Indications, benefits, and risks of Pilates exercise for people with chronic low back pain: a Delphi survey of Pilates-trained physical therapists. Braz. J. Phys. Ther. 2014;94 (6):806-817.

13. Duarte DDS, Sousa CAD, Nunes CRDO. Effect of Pilates method and conversation circles on the health of older adults. Fisioter. Mov. 2017;30 (1):39-48.

14. Guimarães ACDA, Azevedo SFD, Simas JPN, Machado Z, Jonck VTF. The effect of Pilates method on elderly flexibility. Fisioter. Mov. 2014;27 (2):181188.

15. Ribeiro IA, Oliveira TD, Blois CR. Effects of Pilates and Classical Kinesiotherapy on chronic low back pain: a case study. Fisioter. Mov. 2015;28 (4):759-765.

16. Wajswelner H, Metcalf B, Bennell K. Clinical Pilates versus General exercise for chronic low back pain: Randomized trial. Med Sci Sports Exerc. 2012;44 (7):1197-1205.

17. Yamato TP, Maher CG, Saragiotto BT, Hancock MJ, Ostelo RW, Cabral CM et al. Pilates for low back pain: complete republication of a cochrane review. Spine. 2016;41 (12):1013-1021. 
18. Galdino II, De Carvalho FT, de Carvalho MEIM, Serafim GML, do Nascimento, TSM. Efeitos do Método Pilates em pacientes com lombalgia crônica inespecífica: revisão sistemática. Rics. 2015;2 (3):122-129.

19. Nogueira TRB, de Oliveira GL, Perini-de-Oliveira TA, Pagani MM, Valentim-Silva JR. Efeitos do Método Pilates nas adaptações neuromusculares e na composição corporal de adultos jovens. Rbpfex. 2014;8 (45):296-303.

20. Guclu-Gunduz A, Citaker S, Irkec C, Nazliel B, Batur-Caglayan HZ. The effects of Pilates on balance, mobility and strength in patients with multiple sclerosis. NeuroRehabilitation. 2014;34 (2):337-342
21. Tomruk MS, Uz MZ, Kara B, \diman E. Effects of Pilates exercises on sensory interaction, postural control and fatigue in patients with multiple sclerosis. Multiple sclerosis and related disorders. 2016;7 (3):70-73.

22. Angin E, Erden Z, Can F. The effects of clinical Pilates exercises on bone mineral density, physical performance and quality of life of women with postmenopausal osteoporosis. J Back Musculoskelet Rehabil. 2015;28 (4):849-858.

23. Bird ML, Fell J. Positive long-term effects of Pilates exercise on the age-related decline in balance and strength in older, community-dwelling men and women. J Aging Phys Act. 2014;22 (3):342-347. 